\title{
STRONGLY IRRESOLVABLE SPACES
}

\author{
DAVID ROSE, KARI SIZEMORE, AND BEN THURSTON
}

Received 9 March 2006; Revised 31 May 2006; Accepted 5 June 2006

Several new characterizations of strongly irresolvable topological spaces are found and precise relationships are noted between strong irresolvability, hereditary irresolvability, and submaximality. It is noted that strong irresolvablity is a faint topological property, while neither hereditary irresolvablity nor submaximality are semitopological.

Copyright (c) 2006 Hindawi Publishing Corporation. All rights reserved.

\section{Introduction}

Throughout, $(X, \tau)$ denotes a topological space and if $A \subseteq X, \tau \mid A$ is the subspace topology on $A$. Also, $\mathrm{Cl}(A)$ and $\operatorname{Int}(A)$ denote the closure and interior, respectively, of $A$ in $X$. If $B \subseteq A, \mathrm{Cl}_{A}(B)$ and $\operatorname{Int}_{A}(B)$ are the closure and interior, respectively, of $B$ in the subspace $A$. A subset $D$ of $X$ is dense if $\operatorname{Cl}(D)=X$ and the family of all dense subsets of $X$ is $D(X, \tau)$. A subset $C$ of $X$ is codense if $X-C=D$ is dense, or equivalently, $\operatorname{Int}(C)=\varnothing$. A subset $N$ of $X$ is nowhere dense if $\operatorname{Int}(\mathrm{Cl}(N))=\varnothing$ and the family of nowhere dense subsets of $X$ is $N(X, \tau)$. It is known that a subset $N$ of $X$ is nowhere dense if and only if for each nonempty open set $U$, there exists a nonempty open subset $V \subseteq U$ with $V \cap N=\varnothing$. Further, $N(X, \tau)$ is an ideal. That is, every subset of a nowhere dense set is nowhere dense and every finite union of nowhere dense sets is nowhere dense. This latter property does not hold generally for codense sets. In fact, many spaces exist which are the union of two codense subsets. Equivalently, there exist spaces containing two disjoint dense subsets.

Definition 1.1. A topological space is resolvable if it contains two disjoint dense subsets. A space is irresolvable if it is not resolvable.

Definition 1.2. A topological space is crowded (or dense in itself) if it has no isolated points.

Since an isolated point must belong to every dense set, the presence of isolated points prevents resolvability. Hewitt [3] observed that all classical topological spaces without isolated points are resolvable. In particular, he proved that all crowded first countable spaces are resolvable. He then constructed crowded spaces which are far from being resolvable. 
Definition 1.3. A topological space is submaximal if every dense subset is open.

Definition 1.4. A space is hereditarily irresolvable if every subspace is irresolvable.

If $D$ is a proper dense subset in a space $(X, \tau), D$ is not dense in the space $(X, \sigma)$ where $\sigma=\tau(X-D)=\tau[D]$ is the smallest expansion of $\tau$ for which $D$ is closed. By a maximal expansion construction, Hewitt was able to construct submaximal spaces and hereditarily irresolvable $T_{1}$-spaces. Anderson [1], also using expansion topologies, found a construction for submaximal connected Hausdorff spaces. Hewitt did not investigate strongly irresolvable spaces which were later mentioned or used in $[2,4]$.

Definition 1.5. A space $(X, \tau)$ is strongly irresolvable if each open subspace is irresolvable.

We avoid expansion topologies to present easy examples of submaximal, hereditarily irresolvable, and strongly irresolvable spaces using ultrafilters and Hewitt's decomposition theorem which states that every space $X$ can be expressed as a disjoint union $F \sqcup G$ of a closed resolvable subspace $F$ with an open hereditarily irresolvable subspace $G$. It can easily be shown that this decomposition is unique for each space $X$.

We offer the following obvious but useful fact and then close this section with a proof of the equivalence of various descriptions of strongly irresolvable spaces that have appeared in the literature.

Proposition 1.6. If $U$ is a nonempty open subspace of a resolvable space, then $U$ is a resolvable subspace.

Theorem 1.7. Strong irresolvability of the space $(X, \tau)$ is equivalent to each of the following.

(1) Every open subspace is irresolvable.

(2) Every dense subset has a dense interior.

(3) Every codense set is nowhere dense.

(4) Every subset is the union of an open set and a nowhere dense set.

Proof. (1) $\Leftrightarrow(2)$. If $D$ is dense and $U=X-\mathrm{Cl}(\operatorname{Int}(D)) \neq \varnothing$, then $U-D \neq \varnothing$ for otherwise, $U \subseteq \operatorname{Int}(D)-\operatorname{Int}(D)=\varnothing$. Also, $U \cap D \neq \varnothing$ since $D$ is dense, and $U \cap D$ is dense in $U$ since $U \subseteq \mathrm{Cl}(U)=\mathrm{Cl}(U \cap D)$. But $\operatorname{Int}_{U}(U \cap D)=\operatorname{Int}(U \cap D)=\varnothing \operatorname{since} \operatorname{Int}(U \cap D) \subseteq$ $U \cap \operatorname{Int}(D)=\varnothing$. Thus, $U-D$ is also dense in $U$ and $U$ is resolvable. This contradiction shows that $X-\mathrm{Cl}(\operatorname{Int}(D))=\varnothing$ and hence $\operatorname{Int}(D)$ is dense.

$(2) \Rightarrow(3) . A$ is codense $\Rightarrow X-A$ is dense $\Rightarrow \operatorname{Int}(X-A)$ is dense $\Rightarrow \operatorname{Cl}(\operatorname{Int}(X-A))=$ $X-\operatorname{Int}(\mathrm{Cl}(A))=X \Rightarrow \operatorname{Int}(\mathrm{Cl}(A))=\varnothing \Rightarrow A$ is nowhere dense.

$(3) \Rightarrow(4) . A \subseteq X \Rightarrow \operatorname{Int}(A)=\varnothing \operatorname{or} \operatorname{Int}(A) \neq \varnothing$. If $\operatorname{Int}(A)=\varnothing, A \in C(X, \tau)=N(X, \tau) \Rightarrow$ $A=\varnothing \cup A$ is the union of an open set with a nowhere dense set. If $\operatorname{Int}(A) \neq \varnothing$, then $\operatorname{Int}(A-\operatorname{Int}(A))=\varnothing \Rightarrow A-\operatorname{Int}(A) \in C(X, \tau)=N(X, \tau) \Rightarrow A=\operatorname{Int}(A) \cup(A-\operatorname{Int}(A))$ is a union of an open set with a nowhere dense set.

$(4) \Rightarrow(1)$. If $U$ is open, nonempty, and resolvable, then $U=U_{1} \sqcup U_{2}$ with each $U_{i}$ dense in $U$. So, $U_{1}=V \cup N$ where $V$ is open and $N$ is nowhere dense. $V=\varnothing \Rightarrow U \subseteq$ $\operatorname{Int}\left(\mathrm{Cl}\left(U_{1}\right)\right)=\operatorname{Int}(\mathrm{Cl}(N))=\varnothing$. So, $V \neq \varnothing \Rightarrow \operatorname{Int}_{U}\left(U_{1}\right)=\operatorname{Int}\left(U_{1}\right) \neq \varnothing \Rightarrow U_{2} \cap \operatorname{Int}\left(U_{1}\right) \neq$ $\varnothing \Rightarrow U_{1} \cap U_{2} \neq \varnothing$. This contradiction shows that $U$ is irresolvable. 


\section{Levels of irresolvability}

Theorem 2.1. For any space $(X, \tau)$, submaximality $\Rightarrow$ hereditary irresolvability $\Rightarrow$ strong irresolvability $\Rightarrow$ irresolvability.

Proof. If $X$ is submaximal and $A$ is a nonempty resolvable subspace with $A=A_{1} \sqcup A_{2}$ and each $A_{i}$ is dense in $A$, then $X-A_{2}=(X-A) \cup A_{1}$ is dense in $X$ and hence open. So, $A_{2}$ is closed and $A \subseteq \mathrm{Cl}\left(A_{2}\right)=A_{2} \Rightarrow A_{1}=\varnothing \Rightarrow A=\varnothing$ since $A \subseteq \mathrm{Cl}\left(A_{1}\right)$. This contradiction shows that $X$ is hereditarily irresolvable. If $X$ is hereditarily irresolvable, all subspaces including the open ones are irresolvable so that $X$ is strongly irresolvable. Certainly strongly irresolvable spaces are irresolvable.

Examples will be used to show that these classes of spaces are nonempty and the implications of this theorem are not reversible. Finite spaces can be used for this purpose but these spaces can only be irresolvable if isolated points are present. In fact, Hewitt showed that first countable crowded spaces are always resolvable. The class of first countable spaces includes all finite spaces and all metric spaces. Thus, our examples of irresolvable spaces without isolated points cannot be first countable. The basic unit of construction in these examples is an infinite set $X$ equipped with a free ultrafilter topology $F^{*}=F \cup\{\varnothing\}$, where $F$ is a free ultrafilter on $X$. It is easy to see that every infinite set supports a free ultrafilter. For if $X$ is infinite and $(P, \subseteq)$ is the poset of all filters on $X$, by Zorn's lemma, there is a maximal filter $F$ in $P$, called an ultrafilter, containing the free filter of cofinite subsets of $X$. Clearly $F$ is free and $F^{*}$ is a topology on $X$. Ultrafilters are known to have the following interesting properties.

Proposition 2.2. If $F$ is an ultrafilter on $X$ and $B \subseteq X$ has the property that $B \cap A \neq \varnothing$ for each $A \in F$, then $B \in F$.

Proposition 2.3. If $F$ is an ultrafilter on $X$, then for all subsets $A \subseteq X, A \notin F \Rightarrow A^{\prime}=$ $X-A \in F$.

Proposition 2.4. If $X$ is an infinite set, $F$ is a free ultrafilter on $X$ and $\tau=F^{*}$, then $(X, \tau)$ is a crowded submaximal $T_{1}$-space.

Proof. If $D$ is dense in $X$, then $D \cap A \neq \varnothing$ for each $A \in \tau-\{\varnothing\}=F \Rightarrow D \in F \subseteq \tau$ so that $X$ is submaximal. If $\{x\} \in F$, then for all $A \in F,\{x\} \cap A \neq \varnothing \Rightarrow x \in A$ so $x \in \cap F$ which contradicts the fact that $F$ is free. So, $X$ is crowded. Further $X$ is $T_{1}$ because each $\{x\} \in \tau^{c}$. To see this, note that $\{x\} \notin F \Rightarrow\{x\}^{\prime}=X-\{x\} \in F \subseteq \tau$.

In fact, the space of this proposition is hyperconnected in the sense that every nonempty open set is dense, for if $A \in F$ and $B \in \tau-\{\varnothing\}=F$, then $B \cap A \neq \varnothing \Rightarrow A$ is dense. So, $D(X, \tau)=F \Rightarrow F^{c}=C(X, \tau)=N(X, \tau)$ since submaximal spaces are strongly irresolvable. The irresolvability of this space is strongly dependent on the ultrafilter property of $F$. If $F$ were replaced by simply a free filter, the space might be resolvable. For example, if $X$ is any infinite set and $\rho$ is the cofinite topology on $X$, then the infinite subsets are dense and since two disjoint infinite subsets can be found, the space is resolvable. It is also crowded and $T_{1}$ with $N(X, \rho)$ is the collection of finite subsets. In particular, if $X=N$ is the set of natural numbers, the finite sets are the nowhere dense sets and $E=\{2,4, \ldots\}$ and $O=\{1,3, \ldots\}$ are disjoint dense subsets.

We now construct a hereditarily irresolvable space which fails to be submaximal. 
Example 2.5. Let $Y$ and $Z$ be disjoint infinite sets. Let $F$ be a free ultrafilter on $Y$ and let $G$ be a free ultrafilter on $Z$. Equip $Y$ and $Z$ with topologies $\sigma$ and $\rho$, respectively, with $\sigma=F \cup\{\varnothing\}$ and $\rho=G \cup\{\varnothing\}$. Let $X=Y \sqcup Z$ have topology $\tau=\{A \sqcup B \mid A \in F$ and $B \in$ $\rho\} \cup\{\varnothing\}$. We claim that $(X, \tau)$ is a crowded $T_{1}$-space which is hereditarily irresolvable but not submaximal. First, we see that $\tau$ is a topology. $A_{1}, A_{2} \in F$ and $B_{1}, B_{2} \in \rho \Rightarrow\left(A_{1} \sqcup B_{1}\right) \cap$ $\left(A_{2} \sqcup B_{2}\right)=\left(A_{1} \cap A_{2}\right) \sqcup\left(B_{1} \cap B_{2}\right) \in \tau$ since $A_{1} \cap A_{2} \in F$ and $B_{1} \cap B_{2} \in \rho$. So, $\tau$ is closed under finite intersection. For closure under arbitrary union, let $A_{i} \in F$ and $B_{i} \in \rho$ for each $i \in I$. Then, $\bigcup_{i \in I}\left(A_{i} \sqcup B_{i}\right)=\varnothing \in \tau$ if $I=\varnothing$ and otherwise, $\bigcup_{i \in I}\left(A_{i} \sqcup B_{i}\right)=\left(\bigcup_{i \in I} A_{i}\right) \sqcup$ $\left(\bigcup_{i \in I} B_{i}\right) \in \tau$ since $A_{i_{0}} \subseteq \bigcup_{i \in I} A_{i}$ for some $i_{0} \in I \Rightarrow \bigcup_{i \in I} A_{i} \in F$ and since $\bigcup_{i \in I} B_{i} \in \rho$. To see that each $\{x\}$ is closed, note that if $x \in Y, Y-\{x\} \in F$ and $Z \in G \Rightarrow(Y-\{x\}) \sqcup Z \in$ $\tau \Rightarrow\{x\} \in \tau^{c}$. And $x \in Z \Rightarrow Z-\{x\} \in G \Rightarrow Y \sqcup(Z-\{x\}) \in \tau \Rightarrow\{x\} \in \tau^{c}$. So, $(X, \tau)$ is a $T_{1}$-space. Also, $(X, \tau)$ is crowded since $U \in \tau-\{\varnothing\} \Rightarrow U=A \sqcup B$ for some $A \in F \Rightarrow U$ is infinite. (i.e., $(X, \tau)$ has inifinite dispersion character.) To show that $X$ is hereditarily irresolvable, let $H \subseteq X$ be any subspace. If $H \cap Y=\varnothing$, then $H \subseteq Z \Rightarrow H$ is irresolvable since $\tau \mid Z=\rho$ implies that the subspace $Z$ is hereditarily irresolvable. Otherwise, $H \cap$ $Y \neq \varnothing$ and since $Y=Y \sqcup \varnothing \in \tau, H \cap Y \in \tau \mid H-\{\varnothing\}$. If $H$ were resolvable, then the open set $H \cap Y$ would be resolvable. But, $H \cap Y$ is irresolvable being a subspace of $Y$, a hereditarily irresolvable space. Again, note that $(Y, \tau \mid Y)=(Y, \sigma)$ since $\tau \mid Y=\sigma$. In either case, $H$ is irresolvable so that $(X, \tau)$ is hereditarily irresolvable. We now will show that $X$ is not submaximal. Note now that $Y$ is dense in $X$. For every $A \sqcup B \in \tau-\{\varnothing\}$ has $(A \sqcup B) \cap Y=A \neq \varnothing$ since $A \in F$. Thus, for any $x \in Z, Y \subseteq Y \sqcup\{x\} \Rightarrow Y \sqcup\{x\}$ is dense. But, $Y \sqcup\{x\} \notin \tau$ since $\{x\} \notin \rho$.

In this last example, had $Z$ been equipped with a free filter topology for which $Z$ is resolvable, the construction of $X$ as above results in a strongly irresolvable space which is not hereditarily irresolvable. This is our next example.

Example 2.6. Let $Y$ and $Z$ be disjoint infinite sets. Let $F$ be a free ultrafilter on $Y$. Equip $Y$ with the ultrafilter topology $\sigma=F \cup\{\varnothing\}$ and let $\rho$ be the cofinite topology on $Z$. Let $X=Y \sqcup Z$ have topology $\tau=\{A \sqcup B \mid A \in F$ and $B \in \rho\} \cup\{\varnothing\}$. We claim that $(X, \tau)$ is a crowded $T_{1}$-space which is strongly irresolvable but not hereditarily irresolvable. As in the previous example, $\tau$ is a topology and $(X, \tau)$ is a crowded $T_{1}$-space. Let $U \in \tau-\{\varnothing\}$. Then, $U=A \sqcup B$ with $A=U \cap Y \in F$. Since $Y \in \tau, A \in \tau \mid U$. But also, $A$ is irresolvable as a subspace of $Y \Rightarrow U$ is irresolvable. So, $X$ is strongly irresolvable. But, $Z$ is a resolvable subspace of $X$ implying that $X$ is not hereditarily irresolvable.

The spaces constructed as disjoint unions of spaces with filter topologies in the two examples just given are connected. By disconnecting the two components in the previous example, we obtain the following example of an irresolvable space which is not strongly irresolvable.

Example 2.7. Let $Y$ and $Z$ be infinite sets, let $\sigma=F \cup\{\varnothing\}$ for some free ultrafilter $F$ on $Y$, and let $\rho$ be the cofinite topology on $Z$. Let $X=Y \sqcup Z$ have topology $\tau=\{A \sqcup B \mid$ $A \in \sigma$ and $B \in \rho\}$. As in the previous examples, $\tau$ is a topology and $(X, \tau)$ is a crowded $T_{1}$-space. The space $X$ is irresolvable since $Y$ is an open irresolvable subspace. But $X$ is not strongly irresolvable since $Z$ is an open resolvable subspace. The space $X$ is disconnected since $Z$ is a nonempty proper clopen subset. 


\section{Local resolvability and decomposition of irresolvability levels}

Hewitt's paper [3, Theorem 20] states that a space is resolvable if and only if every nonempty open subset contains a subset which is resolvable as a subspace. He proved this by transfinite induction. One immediate consequence is that a space is resolvable if and only if each nonempty basic open subset contains a resolvable subspace.

Corollary 3.1. A space $(X, \tau)$ is resolvable if and only if there is an open cover of $X$ consisting of resolvable subspaces.

Proof. If $X=\bigcup_{i \in I} U_{i}$ with each $U_{i}$ an open resolvable subspace of $X$, and if $\varnothing \neq W \subseteq X$ is open, then $W \cap U_{j} \neq \varnothing$ for some $j \in I$, and by Hewitt's result there is a resolvable subspace $A \subseteq W \cap U_{j} \subseteq W$. So, $X$ is resolvable. Conversely, if $X$ is resolvable, then $\{X\}$ is an open cover consisting of resolvable subspaces. Moreover, every open cover of $X$ consists of resolvable subspaces since open subspaces of resolvable spaces are resolvable.

Definition 3.2. A space $(X, \tau)$ is resolvable at a point $x \in X$ if $x \in U \in \tau$ for some resolvable $U$. Let $R(\tau)=\{x \in X \mid(X, \tau)$ is resolvable at $x\}$.

It is clear that $R(\tau) \in \tau$ and that $R(\tau)$ is the union of all open resolvable subspaces of $X$. So, $X$ is resolvable if and only if $R(\tau)=X$. That is, locally resolvable spaces are resolvable. In fact, since Hewitt showed that the closure of a resolvable subspace is resolvable, we have that either $R(\tau)=X$ or $\mathrm{Cl}(R(\tau)) \neq X$. In this latter case, $X$ is irresolvable. We have the following observation.

Proposition 3.3. For a space $(X, \tau)$, the following are equivalent.

(1) $X$ is strongly irresolvable.

(2) $R(\tau)=\varnothing$.

(3) $\operatorname{Int}(F)=\varnothing$ if $X=F \sqcup G$ is the Hewitt decomposition with $F$ closed and resolvable.

(4) $X$ has an open hereditarily irresolvable dense subspace.

(5) Every resolvable subspace is nowhere dense.

Proof. $(1) \Rightarrow(2)$. Clear since $R(\tau)$ is open and resolvable if nonempty.

$(2) \Rightarrow(3)$. Also clear since $x \in \operatorname{Int}(F) \Rightarrow x \in R(\tau)$.

(3) $\Leftrightarrow(4)$. Int $(F)=\varnothing \Rightarrow G$ is an open dense hereditarily irresolvable subspace. And, if $H$ was an open dense hereditarily subspace of $X=F \sqcup G$, then $H \cap F=\varnothing$. For otherwise, this subset would be both resolvable, being an open subspace of $F$, and irresolvable. Thus, $H \subseteq G \Rightarrow G$ is dense in $X$ so that $\operatorname{Int}(F)=\varnothing$.

(3) $\Rightarrow(5)$. If $A$ is resolvable then $\operatorname{Cl}(A)$ is resolvable. If $A$ is not nowhere dense, then $\operatorname{Int}(\mathrm{Cl}(A))$ is open, nonempty, and resolvable which implies that $\operatorname{Int}(\operatorname{Cl}(A)) \cap G=\varnothing$. Thus, $\operatorname{Int}(F) \neq \varnothing$ which contradicts $F$ being nowhere dense.

$(5) \Rightarrow(1)$. Clear since nonempty open subsets are not nowhere dense.

Definition 3.4. A space $(X, \tau)$ is homogeneous if for any pair $x, y$ of distinct points, there is a homeomorphism $h: X \rightarrow X$ with $y=h(x)$.

Topological groups are homogeneous spaces. 
Proposition 3.5. If $(X, \tau)$ is homogeneous, then $X$ is strongly irresolvable if and only if $X$ is irresolvable.

Proof. Either $R(\tau)=\varnothing$ or $R(\tau) \neq \varnothing$. If $R(\tau) \neq \varnothing$, by homogeneity, $R(\tau)=X$.

Evidently, homogeneity plus irresolvability implies strong irresolvability. But, this does not constitute a decomposition of strong irresolvability since homogeneity is not implied by strong irresolvability.

We introduce three conditions that a space $(X, \tau)$ may have

(i) $C_{1}$ : every proper regular open set is irresolvable,

(ii) $C_{2}$ : every nowhere dense set is irresolvable,

(iii) $C_{3}$ : every irresolvable nowhere dense set is closed.

We also label the levels of irresolvability as follows:

(i) $I$ : The space is irresolvable,

(ii) SI: the space is strongly irresolvable,

(iii) HI: the space is hereditarily irresolvable,

(iv) $S$ : the space is submaximal.

If a label of a property is enclosed in brackets, the class of all spaces having the property is intended. For example, $\mathrm{SI} \Rightarrow C_{1}$ can also be indicated by $[\mathrm{SI}] \subseteq\left[C_{1}\right]$. The equation $\mathrm{SI}=C_{1}+I$ denotes a decomposition of strong irresolvability into the join of two strictly weaker conditions, that is, $[\mathrm{SI}]=\left[C_{1}\right] \cap[I]$ and $[\mathrm{SI}] \varsubsetneqq\left[C_{1}\right]$ and $[\mathrm{SI}] \varsubsetneqq[I]$. An earlier example showed that $I \nRightarrow$ SI. The following example shows that $C_{1} \nRightarrow$ SI. It also shows that $C_{2} \nRightarrow \mathrm{HI}$, and the same example shows that $C_{3} \nRightarrow S$.

Example 3.6. Let $(N, \rho)$ be the set of natural numbers with the cofinite topology. Then $N$ is a crowded resolvable $T_{1}$-space and it has property $C_{1}$. For if $U$ is a nonempty regular open set, $U=\operatorname{Int}(\mathrm{Cl}(U))=\operatorname{Int}(X)=X$. Thus, $N$ does not have any nonempty proper regular open subset. So, $N$ has $C_{1}$ but not $I$ and hence neither SI nor HI. This space also has $C_{2}$, for the nowhere dense sets are the finite subsets. Since infinite sets are dense and if a set $F$ is finite, it is closed in the cofinite topology and has empty interior. Also the subspace $(F, \rho \mid F)$ is discrete and every point is an isolated point in the subspace. For if $x \in F$, then $U=N-(F-\{x\}) \in \rho$ and $U \cap F=\{x\} \in \rho \mid F$. So, the subspace $F$ is irresolvable. Since all nowhere dense sets are irresolvable and all nowhere dense sets are also closed, $C_{3}$ is satisfied as well.

Theorem 3.7. $\mathrm{SI}=C_{1}+I$.

Proof. Clearly, SI $\Rightarrow C_{1} \wedge I$. For the reverse implication, let $X=F \sqcup G$ be the Hewitt decomposition of an irresolvable space with $F$ closed and resolvable. Then, if $U=\operatorname{Int}(F) \neq$ $\varnothing, U$ is a nonempty proper regular open subset since $\operatorname{Int}(\mathrm{Cl}(U))=\operatorname{Int}(\mathrm{Cl}(\operatorname{Int}(\mathrm{Cl}(F))))=$ $\operatorname{Int}(\mathrm{Cl}(F))=\operatorname{Int}(F)=U$. By property $C_{1}, U$ is irresolvable and this contradicts the resolvability of $F$. So, $\operatorname{Int}(F)=\varnothing$ and, thus, $(X, \tau)$ is SI.

Theorem 3.8. $\mathrm{HI}=\mathrm{SI}+C_{2}$.

Proof. Clearly, $\mathrm{HI} \Rightarrow \mathrm{SI} \wedge C_{2}$. For the reverse implication, let $(X, \tau)$ be a $C_{2} \wedge$ SI space and let $X=F \sqcup G$ be the Hewitt decomposition. Then, $G$ is a nonempty open hereditarily 
irresolvable subspace and $\operatorname{Int}(F)=\varnothing$. It follows that $F$ is nowhere dense and resolvable if nonempty. Evidently by $C_{2}, F=\varnothing$ and $X=G$ is $\mathrm{HI}$.

Theorem 3.9. $S=\mathrm{HI}+C_{3}$.

Proof. Certainly, $S \Rightarrow C_{3} \wedge$ HI. For the reverse implication let $(X, \tau)$ be a $C_{3} \wedge$ HI space and let $D$ be dense in $X$. Then $X-D$ is codense and hence nowhere dense and irresolvable subspace. By $C_{3}, X-D$ is closed and $D$ is open.

It might be worth noting that the $\alpha$-space property, $X=X^{\alpha}$, has a decomposition $C_{2}+$ $C_{3}$. The $\alpha$-space for the space $(X, \tau)$ is $X^{\alpha}=\left(X, \tau^{\alpha}\right)$, where $\tau^{\alpha}=\{U-E \mid U \in \tau$ and $E \in$ $N(X, \tau)\}$ is the smallest expansion of $\tau$ for which all $\tau$-nowhere dense sets are closed [8]. Clearly, $X=X^{\alpha}$ if and only if $C_{2} \wedge C_{3}$. It only remains to see that neither $C_{2}$ nor $C_{3}$ alone implies $X=X^{\alpha}$. In the example given earlier of a HI space which is not $S$, clearly $C_{2}$ holds but not $C_{3}$ for otherwise, so would $S$.

Example 3.10. Let $Y=\{0\} \cup N$ have topology $\sigma=\{\varnothing,\{0\}\} \cup\{V \subseteq Y \mid 0 \in V$ and $Y-$ $V$ is finite $\}$. Then $E=\{2,4,6, \ldots\}$ is a resolvable nowhere dense set. $D_{1}=\{2+4 n\}$ and $D_{2}=\{4(1+n)\}$ are disjoint dense subsets of $E$. But $E$ is not closed since $\mathrm{Cl}(E)=N$. So, $Y$ does not have $C_{2}$. However, if $F$ is an irresolvable nowhere dense subset of $Y$, then $F$ is codense $\Rightarrow 0 \notin F$. But, then to be irresolvable, $F$ must be finite and hence closed. Thus, $Y$ has $C_{3}$.

\section{Finite products}

A basic problem that remained unsolved for several decades following Hewitt's discovery of irresolvable spaces was the question of irresolvability of finite product spaces. A property is said to be finitely productive if the product space $X \times Y$ has the property whenever both factor spaces $X$ and $Y$ have the property. It was incorrectly stated in [2], that strong irresolvability is finitely productive. This claim is strongly negated by the following simple counterexample.

Example 4.1. Let $X$ be an infinite set, let $F$ be a free ultrafilter on $X$, and equip $X$ with the topology $\tau=F \cup\{\varnothing\}$. Then $X$ is a crowded submaximal $T_{1}$-space. Let $X^{2}=X \times X$ have the product topology $\pi$ and let $D=\{(x, x) \mid x \in X\} \subseteq X^{2}$ be the diagonal subset. We will show that $D$ is dense and codense in $X^{2}$. Thus $X=D \sqcup(X-D)$ is resolvable being a disjoint union of dense sets. To see that $D$ is dense, note that every nonempty open set $W \in \pi$ contains a nonempty basic open set $U \times V \subseteq W$. Since $U \neq \varnothing$ and $V \neq \varnothing$, we have $U, V \in F$. So, $U \cap V \in F \Rightarrow U \cap V \neq \varnothing$. If $x \in U \cap V,(x, x) \in(U \times V) \cap D \Rightarrow$ $W \cap D \neq \varnothing$ and hence $D$ is dense. Also, $\operatorname{Int}(D)=\varnothing$. For if $U \times V$ is a nonempty basic open set, then $U, V \in F \Rightarrow U$ and $V$ are infinite sets and if $x \in U$, there exists $y \in V$ with $y \neq x$. Then $(x, y) \in(U \times V)-D$. So, $U \times V \nsubseteq D$. Evidently, $D$ is codense and $X-D$ is also dense.

Malyhin showed in [6] that any infinite sets $X$ and $Y$ have topologies for which these spaces are irresolvable $T_{1}$-spaces and yet the product space $X \times Y$ is maximally resolvable. Maximal resolvability requires that the number of pairwise disjoint dense subsets 
that exist equals the least number of elements in a nonempty open set, which is the dispersion character. Malyhin also showed that if a free ultrafilter exists with the property that countably infinite intersections of its members are still members, then $T_{1}$-spaces exist whose product is irresolvable. Malyhin [7] has shown that it is consistent with ZFC (Zermelo-Frankel + choice) set theory that all finite products of infinite crowded spaces are resolvable.

\section{Nearly open sets}

Definition 5.1. A subset $A$ of a space $(X, \tau)$ is almost open if $A \subseteq \operatorname{Int}(\mathrm{Cl}(A))$ and $A$ is semiopen if $A \subseteq \mathrm{Cl}(\operatorname{Int}(A))$. The collection of all almost open subsets is denoted $\operatorname{AO}(X, \tau)$ and $\mathrm{SO}(X, \tau)$ denotes the family of semiopen subsets of $(X, \tau)$.

It is easy to show that $\mathrm{AO}(X, \tau)$ and $\mathrm{SO}(X, \tau)$ are closed under arbitrary union but not generally under arbitrary intersection. It is also known that $\tau^{\alpha}=\operatorname{AO}(X, \tau) \cap \operatorname{SO}(X, \tau)$. Further, $A \in \operatorname{AO}(X, \tau)$ if and only if $A=U \cap D$ for some $U \in \tau$ and some dense subset $D$. Note that every dense set $D$ is almost open. For any set $A \subseteq X$, the AO-interior of $A$ is $\operatorname{Int}_{\mathrm{AO}}(A)=\cup\{B \subseteq A \mid B \in \mathrm{AO}(X, \tau)\}$ and the SO-interior of $A$ is $\operatorname{Int}_{\mathrm{SO}}(A)=$ $\cup\{B \subseteq A \mid B \in \mathrm{SO}(X, \tau)\}$. It is known that $\operatorname{Int}_{\mathrm{AO}}(A)=A \cap \operatorname{Int}(\mathrm{Cl}(A))$ and $\operatorname{Int}_{\mathrm{SO}}(A)=$ $A \cap \mathrm{Cl}(\operatorname{Int}(A))$.

Definition 5.2. A subset $A$ of a space $(X, \tau)$ is faintly open if either $A=\varnothing \operatorname{or} \operatorname{Int}(A) \neq \varnothing$. The collection of faintly open sets is $\operatorname{FO}(X, \tau)$.

The nonempty faintly open sets are the noncodense sets. Evidently, complements of nonempty faintly open sets cannot be dense. In particular, a set is dense if and only if it intersects nonemptily every nonempty faintly open set.

Proposition 5.3. A space $(X, \tau)$ is irresolvable if and only if every dense set is faintly open. That is, $D(\tau) \subseteq \mathrm{FO}(X, \tau)$.

Proof. A space is irresolvable if and only if no dense set is codense.

Definition 5.4. A subset $D$ is $\mathrm{AO}$-dense if every nonempty $A \in \operatorname{AO}(X, \tau)$ has $A \cap D \neq \varnothing$. The smallest topology containing $\mathrm{AO}(X, \tau)$ is $\tau_{A}=\langle\operatorname{AO}(X, \tau)\rangle . D$ is $\tau_{A}$-dense if every nonempty $U \in \tau_{A}$ has $U \cap D \neq \varnothing$.

Lemma 5.5. A set $D$ is $\mathrm{AO}-$ dense if and only if $D \cup \mathrm{Cl}(\operatorname{Int}(D))=X$.

Proof. It is known that the union of all almost open subsets of $X-D$ is $(X-D) \cap$ $\operatorname{Int}(\mathrm{Cl}(X-D))$. If $D$ is $A O$-dense, $(X-D) \cap \operatorname{Int}(\mathrm{Cl}(X-D))=\varnothing$ so that $X=D \cup$ $\mathrm{Cl}(\operatorname{Int}(D))$.

THeOREM 5.6. The following are equivalent for a space $(X, \tau)$.

(1) $X$ is strongly irresolvable.

(2) $\mathrm{AO}(X, \tau) \subseteq \mathrm{SO}(X, \tau)$.

(3) $\mathrm{AO}(X, \tau) \subseteq \mathrm{FO}(X, \tau)$.

(4) Every dense set is AO-dense.

(5) Every dense set is $\tau_{A}$-dense.

(6) $X^{\alpha}$ is submaximal. 
Proof. (1) $\Rightarrow(2)$. If $(X, \tau)$ is SI and $A=U \cap D \in \mathrm{AO}(X, \tau)$ for some $U \in \tau$ and dense $D$, then $\operatorname{Int}(D)$ is dense and $\mathrm{Cl}(U \cap \operatorname{Int}(D))=\mathrm{Cl}(U)$ so that $U \cap D \subseteq U \subseteq \mathrm{Cl}(\operatorname{Int}(U \cap$ $\operatorname{Int}(D))) \subseteq \mathrm{Cl}(\operatorname{Int}(U \cap D))$. Thus, $A \in \mathrm{SO}(X, \tau)$.

$(2) \Rightarrow(3)$. This is clear since $\mathrm{SO}(X, \tau) \subseteq \mathrm{FO}(X, \tau)$.

$(3) \Rightarrow(4)$. This is clear since every nonempty faintly open set has nonempty interior which must then intersect every dense set.

$(4) \Rightarrow(1)$. If $D$ is dense in $(X, \tau)$ then $D$ is AO-dense and by Lemma 5.5, $X=D \cup$ $\mathrm{Cl}(\operatorname{Int}(D))$. Then if $\operatorname{Int}(D)$ is not dense, $U=X-\mathrm{Cl}(\operatorname{Int}(D)) \in \tau$ is nonempty and $U \subseteq$ $D \Rightarrow U \subseteq \operatorname{Int}(D)-\operatorname{Int}(D)=\varnothing$. This contradiction shows that $\operatorname{Int}(D)$ is dense and hence $(X, \tau)$ is strongly irresolvable.

$(2) \Rightarrow(5)$. Since $(2) \Rightarrow(4)$, we have that every dense set is AO-dense and since by (2), $\tau^{\alpha}=\mathrm{AO}(X, \tau)$, also $\tau_{A}=\tau^{\alpha}$ and every dense set is $\tau_{A}$-dense.

(5) $\Rightarrow(4)$. This is clear since $\mathrm{AO}(X, \tau) \subseteq \tau_{A}$.

$(1) \Rightarrow(6)$. If $(X, \tau)$ is strongly irresolvable and $D$ is a dense subset of $\left(X, \tau^{\alpha}\right)$, then $D$ is dense in $(X, \tau)$ and $\operatorname{Int}(D)$ is dense in $(X, \tau)$. Since $X-\operatorname{Int}(D)$ is closed and codense, it is nowhere dense. Thus, $X-D \subseteq X-\operatorname{Int}(D) \Rightarrow X-D$ is nowhere dense in $(X, \tau)$. Thus, $X-D$ is closed in $\left(X, \tau^{\alpha}\right)$ and so, $D \in \tau^{\alpha}$.

$(6) \Rightarrow(1)$. If $\left(X, \tau^{\alpha}\right)$ is submaximal and $D$ is dense in $(X, \tau)$, then $D$ is dense in $\left(X, \tau^{\alpha}\right)$ for if $U-E \in \tau^{\alpha}$ for some nonempty $U \in \tau$ and nowhere dense $E$, then $U-\operatorname{Cl}(E) \in \tau$ and nonempty implies $(U-\mathrm{Cl}(E)) \cap D \neq \varnothing$ and thus $(U-E) \cap D \neq \varnothing$. So, $D=V-F$ for some $V \in \tau$ and $F$ nowhere dense. Also, $V-\mathrm{Cl}(F) \in \tau$ and $\mathrm{Cl}(V-\mathrm{Cl}(F))=\mathrm{Cl}(V) \supseteq$ $\mathrm{Cl}(D)=X \Rightarrow \mathrm{Cl}(\operatorname{Int}(D))=X$, so that $\operatorname{Int}(D)$ is dense. Therefore, $(X, \tau)$ is strongly irresolvable.

Proposition 5.7. If $(X, \tau)$ is crowded, then $\left(X, \tau_{A}\right)$ is discrete if and only if $(X, \tau)$ is resolvable.

Proof. Suppose first that $(X, \tau)$ is resolvable and that for some dense set $D, X-D=E$ is also dense. If $x \in X$, then $D^{*}=D \cup\{x\}$ and $E^{*}=E \cup\{x\}$ are dense. So, $D^{*}, E^{*} \in$ $\mathrm{AO}(X, \tau) \subseteq \tau_{A} \Rightarrow\{x\}=D^{*} \cap E^{*} \in \tau_{A}$. Evidently $\left(X, \tau_{A}\right)$ is discrete. Conversely, if $(X, \tau)$ is crowded, let $X=F \sqcup G$ be the Hewitt decomposition. The sketch of the argument is that $\tau_{A} \mid G=(\tau \mid G)_{A}$ since $G \in \tau$. Then, since $(G, \tau \mid G)$ is SI, $(\tau \mid G)_{A}=(\tau \mid G)^{\alpha}$ is a crowded topology since $(G, \tau \mid G)$ is crowded. This is a contradiction unless $G=\varnothing$. Therefore, $X=F$ is resolvable.

Corollary 5.8. If $(X, \tau)$ is crowded and irresolvable, then $\tau_{A}$ is not discrete.

Definition 5.9. A space $X$ is $\mathrm{ED}$ (extremally disconnected) if $\mathrm{Cl}(U)$ is open for every open set $U$.

Proposition 5.10. A space $(X, \tau)$ is $\mathrm{ED}$ if and only if $\mathrm{SO}(X, \tau) \subseteq \mathrm{AO}(X, \tau)$.

Proof. If $\mathrm{SO}(X, \tau) \subseteq \mathrm{AO}(X, \tau)$ and $U \in \tau$, then $\mathrm{Cl}(U) \in \mathrm{SO}(X, \tau) \Rightarrow \mathrm{Cl}(U) \subseteq \operatorname{Int}(\mathrm{Cl}(\mathrm{Cl}(U)))$ $=\operatorname{Int}(\mathrm{Cl}(U)) \Rightarrow \mathrm{Cl}(U) \in \tau$ and hence $(X, \tau)$ is $\mathrm{ED}$. Conversely, if $(X, \tau)$ is $\mathrm{ED}$ and $A \in$ $\mathrm{SO}(X, \tau)$, then $A \subseteq \mathrm{Cl}(\operatorname{Int}(A)) \in \tau \Rightarrow A \subseteq \operatorname{Int}(\operatorname{Cl}(\operatorname{Int}(A))) \subseteq \operatorname{Int}(\mathrm{Cl}(A))$ and $A \in \mathrm{AO}(X, \tau)$. 
In [1] a method is found for constructing connected Hausdorff crowded submaximal spaces. If $(X, \tau)$ is such a space, then $\tau=\tau^{\alpha}=\operatorname{AO}(X, \tau)=\tau_{A}$ and $\operatorname{AO}(X, \tau) \varsubsetneqq \operatorname{SO}(X, \tau)$. This last inequality is forced by the fact that the space is Hausdorff and connected and hence not ED.

\section{Functions and irresolvability}

Definition 6.1. A bijection $f: X \rightarrow Y$ is a faint (semi)homeomorphism if both $f$ and $f^{-1}$ preserve faintly (semi)open sets. A property $P$ transmitted by faint (semi)homeomorphism is a faint (semi)topological property.

It is clear that every semihomeomorphism is a faint homeomorphism so that faint topological properties are semitopological. The following example shows that not every faint homeomorphism is a semihomeomorphism.

Example 6.2. Let $(R, \sigma)$ be the Sorgenfrey line. That is, the set $\{[a, b) \mid a<b\}$ is a base for $\sigma$. Also, let $(R, \tau)$ be the usual space of reals. Then, the identity function $f:(R, \tau) \rightarrow(R, \sigma)$ is a faint homeomorphism but not a semihomeomorphism. For $[0,1]=\mathrm{Cl}_{\tau}((0,1)) \in$ $\mathrm{SO}(R, \tau)$, but $[0,1]=f([0,1]) \nsubseteq[0,1)=\mathrm{Cl}_{\sigma}\left(\operatorname{Int}_{\sigma}([0,1])\right)$ shows that $f([0,1]) \notin \mathrm{SO}(R, \sigma)$, so that $f$ is not a semihomeomorphism. On the other hand $f$ is a faint homeomorphism since each nonempty open interval $(a, b)$ contains a nonempty right open interval $[c, d)$ and vice versa. That is, each topology $\tau$ and $\sigma$ is a $\pi$-base for the other. A $\pi$-base could be called a faint base.

Proposition 6.3. If $f: X \rightarrow Y$ is a bijection, $f$ is a faint homeomorphism if and only if both $f$ and $f^{-1}$ preserve dense sets.

Corollary 6.4. A composition of two faint homeomorphisms is a faint homeomorphism.

Proposition 6.5. Every faint homeomorphism directly and inversely preserves nowhere dense sets.

Proof. If $f: X \rightarrow Y$ is a faint homeomorphism and $E \subseteq X$ is nowhere dense, let $V$ be any nonempty open subset of $Y$. Then, $\operatorname{Int}\left(f^{-1}(V)\right)=U \neq \varnothing$ and so there exists a nonempty open subset $U^{\prime} \subseteq U$ such that $U^{\prime} \cap E=\varnothing$. It follows that $\varnothing \neq V^{\prime}=\operatorname{Int}\left(f\left(U^{\prime}\right)\right) \subseteq V$ and $V^{\prime} \cap f(E)=\varnothing$ showing that $f(E)$ is nowhere dense. So, $f$ and, by symmetry of argument, $f^{-1}$ preserve nowhere dense sets.

It is a corollary that $X$ and $X^{\alpha}$ share the same nowhere dense sets since the identity function $f: X \rightarrow X^{\alpha}$ is a faint homeomorphism.

Let us say that a bijection $f:(X, \tau) \rightarrow(Y, \sigma)$ is an $\alpha$-faint homeomorphism if $f^{\alpha}$ : $\left(X, \tau^{\alpha}\right) \rightarrow\left(Y, \sigma^{\alpha}\right)$ is a faint homeomorphism where for each $x \in X, f(x)=f^{\alpha}(x)$. Then we have the following.

Proposition 6.6. The bijection $f:(X, \tau) \rightarrow(Y, \sigma)$ is a faint homeomorphism if and only if it is an $\alpha$-faint homeomorphism.

Proof. If $i: X \rightarrow X^{\alpha}$ and $j: Y \rightarrow Y^{\alpha}$ are identity maps, then $f^{\alpha}=j \circ f \circ i^{-1}$ is a composition of faint homeomorphisms if $f$ is a faint homeomorphism and $f=j^{-1} \circ f^{\alpha} \circ i$ is a composition of faint homeomorphisms if $f^{\alpha}$ is a faint homeomorphism. 
Proposition 6.7. If $f: X \rightarrow Y$ is a faint homeomorphism, then direct and inverse images under $f$ of nonempty almost open sets contain nonempty almost open sets.

Proof. Suppose that $A \subseteq X$ is almost open and nonempty. Then, $A=U \cap D$ for some nonempty open set $U \subseteq X$ and for some dense subset $D \subseteq X$. Then, $f(A)=f(U) \cap f(D)$. Since $f$ is a faint homeomorphism, $f(D)$ is dense in $Y$ and $V=\operatorname{Int}(f(U)) \neq \varnothing$. Thus, the almost open set $V \cap f(D)$ is a nonempty subset of $f(A)$. The argument for inverse images is the same.

Example 6.8. Let $(R, \tau)$ be the usual space of reals and let $f:(R, \tau) \rightarrow(R, \tau)$ be defined by $f(x)=x$ if $|x| \neq 1$ and $f(x)=-x$ if $|x|=1$. Then $f=f^{-1}$ is a faint homeomorphism which is not a semihomeomorphism since images of open sets may be neither semiopen nor almost open. In particular, $f((-\infty, 0))=(-\infty,-1) \cup(-1,0) \cup\{1\}$ is neither semiopen nor almost open since $1 \notin \mathrm{Cl}(\operatorname{Int}(f((-\infty, 0))))=(-\infty, 0] \supseteq \operatorname{Int}(\mathrm{Cl}(f((-\infty, 0))))$.

It is known [9] that semitopological properties are precisely those properties shared by both $X$ and $X^{\alpha}$. Apparently, both submaximality and hereditary irresolvability are not semitopological but strong irresolvability is semitopological. In fact, more can be said for strong irresolvability.

Theorem 6.9. Strong irresolvability is a faint topological property.

Proof. Let $f: X \rightarrow Y$ be a faint homeomorphism, let $X \neq \varnothing$ be strongly irresolvable, and let $E$ be dense in $Y$. Then $f^{-1}(E)$ is dense in $X$ implying that $\operatorname{Int}\left(f^{-1}(E)\right)$ is dense implying that $E=f\left(f^{-1}(E)\right)$ is faintly open and $\operatorname{Int}(E) \neq \varnothing$. If $\operatorname{Int}(E)$ is not dense, then $\varnothing \neq V=Y-\mathrm{Cl}(\operatorname{Int}(E))$ is open. So, $\operatorname{Int}\left(f^{-1}(V)\right) \neq \varnothing \Rightarrow \operatorname{Int}\left(f^{-1}(V)\right) \cap \operatorname{Int}\left(f^{-1}(E)\right)=$ $\operatorname{Int}\left(f^{-1}(V \cap E)\right) \neq \varnothing \Rightarrow \operatorname{Int}\left(f\left(\operatorname{Int}\left(f^{-1}(V \cap E)\right)\right)\right) \neq \varnothing \Rightarrow \operatorname{Int}(V \cap E)=V \cap \operatorname{Int}(E) \neq \varnothing$ which is a contradiction. So, $\operatorname{Int}(E)$ is dense and $Y$ is strongly irresolvable.

Remark 6.10. It may be noted that submaximality is preserved by open surjections, and hence expansions of submaximal topologies are submaximal.

It was shown in [5] that a space $(X, \tau)$ is submaximal if and only if $\tau=\mathrm{AO}(X, \tau)$. We extend this result slightly.

Proposition 6.11. A space $(X, \tau)$ is submaximal if and only if $\tau=\tau_{A}$.

Proposition 6.12. A space $(X, \tau)$ is strongly irresolvable if and only if $\tau^{\alpha}=\tau_{A}$.

Proof. $X$ is SI if and only if $X^{\alpha}$ is submaximal.

\section{Acknowledgment}

The authors are grateful to the referees for stylistic improvement and a simple example of a faint homeomorphism.

\section{References}

[1] D. R. Anderson, On connected irresolvable Hausdorff spaces, Proceedings of the American Mathematical Society 16 (1965), no. 3, 463-466. 


\section{Strongly irresolvable spaces}

[2] J. Dontchev, M. Ganster, and D. Rose, $\alpha$-scattered spaces. II, Houston Journal of Mathematics 23 (1997), no. 2, 231-246.

[3] E. Hewitt, A problem of set-theoretic topology, Duke Mathematical Journal 10 (1943), no. 2, 309333.

[4] K. Kunen, A. Szymański, and F. Tall, Baire irresolvable spaces and ideal theory, Annales Mathematicae Silesianae (1986), no. 14, 98-107.

[5] R. A. Mahmoud and D. Rose, A note on submaximal spaces and SMPC functions, Demonstratio Mathematica 28 (1995), no. 3, 567-573.

[6] V. I. Malyhin, Products of ultrafilters and irresolvable spaces, Mathematics of the USSR-Sbornik 19 (1973), no. 1, 105-115.

[7] _ On the resolvability of the product of two spaces and a problem of Katertov, Soviet Mathematics-Doklady 16 (1975), 725-729.

[8] O. Njàstad, On some classes of nearly open sets, Pacific Journal of Mathematics 15 (1965), no. 3, 961-970.

[9] D. Rose and T. R. Hamlett, Ideally equivalent topologies and semitopological properties, Mathematical Chronicle 20 (1991), 149-156.

David Rose: Department of Mathematics, Florida Southern College, Lakeland, FL 33801-5698, USA

E-mail address: drose@flsouthern.edu

Kari Sizemore: Department of Mathematics, Florida Southern College, Lakeland, FL 33801-5698, USA

E-mail address: ksizemore@flsouthern.edu

Ben Thurston: Department of Mathematics, Florida Southern College, Lakeland, FL 33801-5698, USA

E-mail address: bthurston@flsouthern.edu 


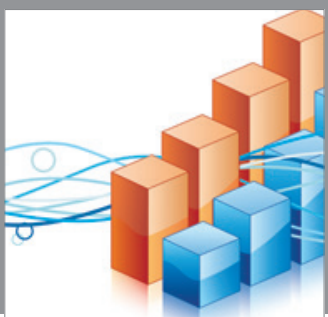

Advances in

Operations Research

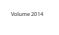

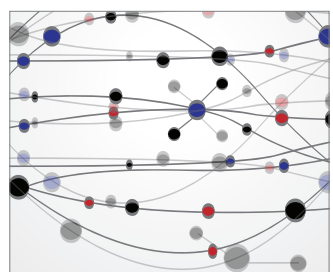

\section{The Scientific} World Journal
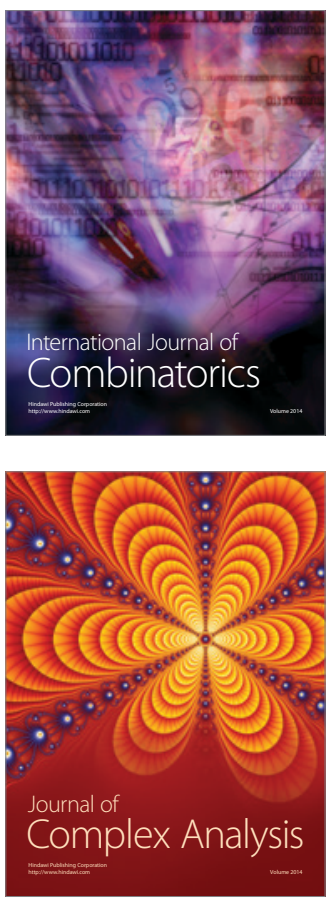

International Journal of

Mathematics and

Mathematical

Sciences
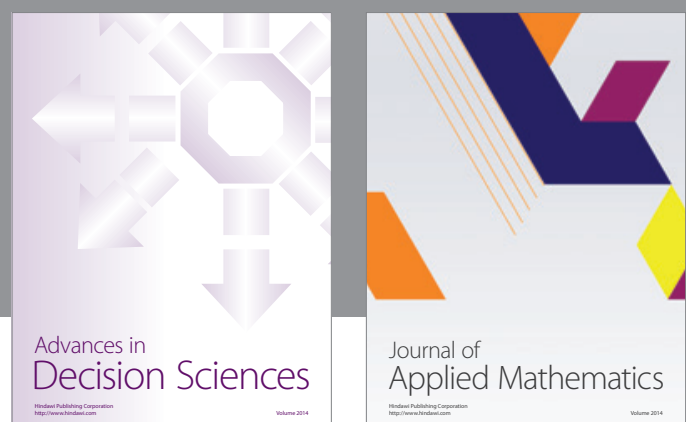

Journal of

Applied Mathematics
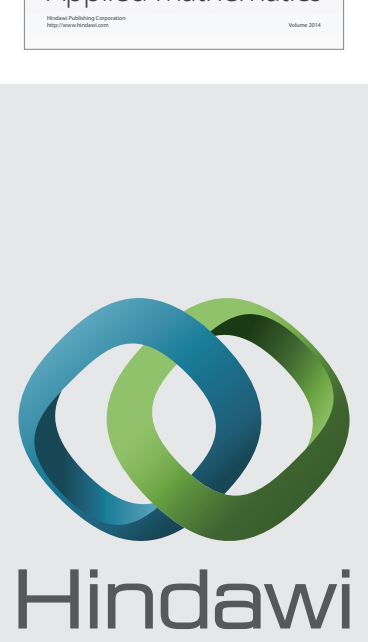

Submit your manuscripts at http://www.hindawi.com
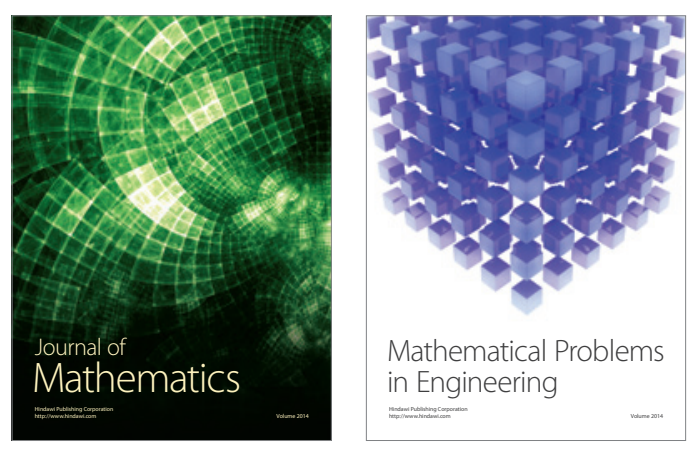

Mathematical Problems in Engineering
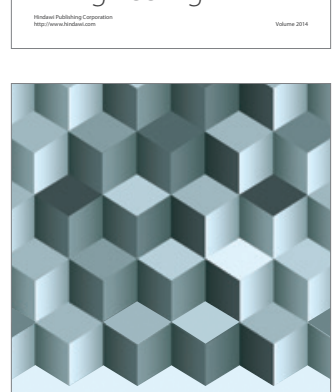

Journal of

Function Spaces
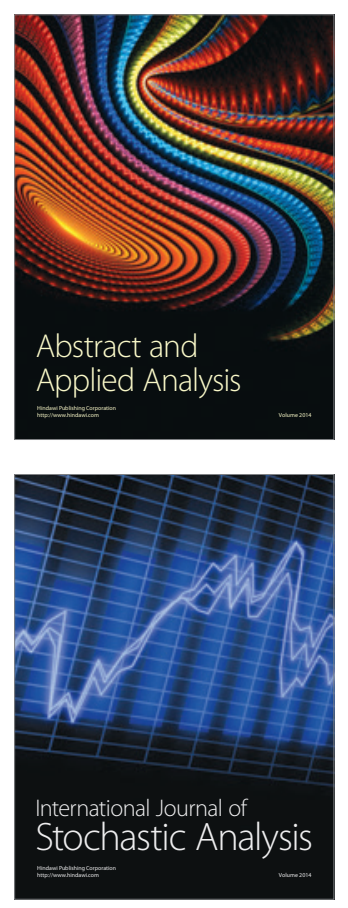

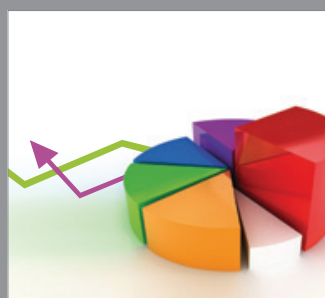

ournal of

Probability and Statistics

Promensencen
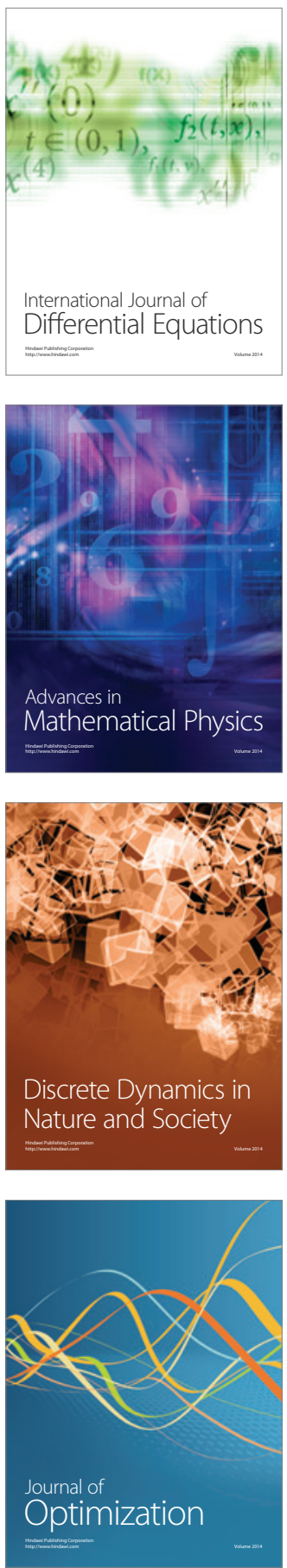